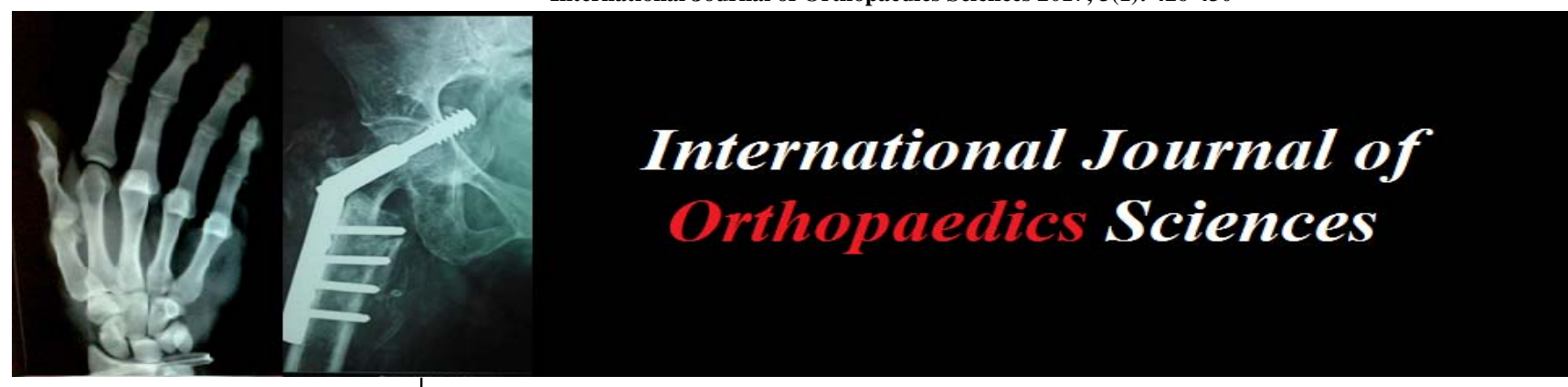

ISSN: $2395-1958$

IJOS 2017; 3(1): 426-430

(C) 2017 IJOS

www.orthopaper.com

Received: 01-11-2016

Accepted: 02-12-2016

Dr. Saubhik Das

M.S. (Ortho), Senior Resident, Department of Orthopaedics, AIIMS, New Delhi, India

Dr. Narendran

M.S. (Ortho), Assistant Professor, Department of Orthopaedics, MGMCRI, Puducherry, India

\section{Dr. Saurabh}

M.S. (Ortho), Senior Resident, Department of Orthopaedics,

VMMC \& Safdarjang Hospital

New Delhi, India

Dr. Navin Kumar Singh M.S. (Ortho), Department of Orthopaedics, AIIMS, New Delhi, India
Correspondence Dr. Narendran M.S. (Ortho), Assistant Professor, Department of Orthopaedics, MGMCRI, Puducherry, India

\section{How efficacious are intra-articular viscosupplements in the management of early osteoarthritis knee?- A detailed comparative study with various outcome measures}

\author{
Dr. Saubhik Das, Dr. Narendran, Dr. Saurabh and Dr. Navin Kumar \\ Singh
}

DOI: http://dx.doi.org/10.22271/ortho.2017.v3.i1f.61

\section{Abstract}

Introduction: Osteoarthritis (OA) knee is the most common debilitating degenerative disorder beyond age 60 seeking medical attention for disabling pain and progressive reduction of joint movements and functional limitation. Conservative and rehabilitative measures remain the mainstay for early stages. In the recent years, intra articular injections particularly steroids and viscosupplementation are increasingly used for early stage management and have raised concerns about their efficacy. Various studies have claimed that intra-articular viscosupplementation with Hyaluronic acid (HA), specially hylan G-F 20 appeared to be safe, effective in pain relief and improved functional outcomes. Having this hypothesis, we wanted to analyze in detail by using various outcome scores (VAS, WOMAC, SF-36) and compare it with the steroids (methyl prednisolone acetate).

Materials and methods: After the institutional review board clearance, this study was conducted as a randomized, prospective study between July 2012 and April 2015. Patients of both sexes beyond age 40 with symptomatic knee OA demonstrating radiological evidence of arthritis [Kellgren-Lawrence (K-L) grade of less than III were randomized into two groups. Patients in the steroid group (group 1, MPG) were subjected to two sittings of intra-articular 6-Methylprednisolone acetate $40 \mathrm{mg}$ each (day 0 and day 7). Patients in viscosupplementation group (group 2, HG) were given a single intra-articular injection of hylan G-F 20. Both groups were given standard physiotherapy regimen post-injection. We used the following parameters to measure outcome, 1) $100 \mathrm{~mm}$ visual analog pain scale (VAS), 2) Western Ontario and McMaster Universities Osteoarthritis index (WOMAC), 3) SF-36 health survey and 4) Range of motion (ROM) to document the baseline characteristics and subsequent follow ups at $2^{\text {nd }}, 4^{\text {th }}$, $12^{\text {th }}, 24^{\text {th }}$ and $52^{\text {nd }}$ week.

Results: Among the 68 enrolled patients, no statistically significant differences were found regarding demographic patterns and the baseline outcome scores of patients between two groups. The mean age, grade and duration at presentation among the two groups were $56.57 / 59.83$ years, grade 2 and $3.4 / 3.8$ years respectively. At 54 weeks, HG group had a mean ROM of $126.93^{\circ}$ compared to $120.83^{\circ}$ in the MPG group $(\mathrm{p}=0.001)$. Regarding VAS, better pain relief $(24.80)$ was noted in the MPG during early period ( $<3$ weeks) as against the HG (67.10). However, reversal of results was noted in the later period $(>3$ months) with the VAS scores doing significantly better in the HG group (18.67) as against MPG (61.18). In the HG group, WOMAC scores revealed a delayed ( $>4$ weeks) onset of improvement, with plateau formation at $6^{\text {th }}$ month (28.43). Early improved WOMAC scores in the MPG showed a decreasing trend reaching pre-injection baseline level (55.93) within 3 months. Post-treatment SF-36 health survey at final follow-up revealed that, patients in hylan group showed greater improvement of mean score from baseline in all 8 functional categories compared to corticosteroid group ( $p$ value $<0.001$ ).

Conclusion: With regard to the intra articular injections in the management of early osteoarthritis, Hylan-G-F-20 has shown a sequential improvement in quality of life beyond 3 weeks post-injection lasting for more than six months. Methylprednisolone acetate gave improved early $(<3$ weeks) response with effect not sustained for more than 3 months.

Keywords: Osteoarthritis, intraarticular injections, hylan-G-F-20, steroids, VAS, WOMAC, SF-36

\section{Introduction}

Osteoarthritis (OA) knee is one of the most common debilitating degenerative disorders seeking medical attention beyond age $60^{[1]}$. The disease process is characterized by progressive degeneration of articular cartilage resulting in narrowing of the joint space, 
osteophyte formation, subchondral cyst, subchondral sclerosis \& synovial inflammation ${ }^{[2]}$. The most important symptom is pain, accompanied by morning stiffness, usually of short duration, reduction of joint movements and progressive functional limitation ${ }^{[3]}$.

Varied treatment options include non pharmacological measures (patient education, loss of weight, bracing, orthotics, physical \& occupational therapy), pharmacological (nonsteroidal anti-inflammatory drugs [NSAID], glucosamine, chondroitin, intra-articular steroids, viscoelastic substances or platelet rich plasmas) \& variety of surgical procedure ranging from arthroscopic debridement to total knee arthroplasty [4]. The goal of the non surgical measures is to eliminate pain, improve movements and delay the need for the replacement procedures by limiting the disability of the patient.

If conventional therapy fails to control symptoms and disability, intra-articular (IA) steroid therapy deserves consideration. Although, it significantly alleviates pain and improves symptoms ${ }^{[5]}$, its long term efficacy, requirement of multiple instillations and its localized [6] and systemic metabolic effects have been debated ${ }^{[7]}$. intra-articular viscosupplementation with Hyaluronic acid (HA), specially hylan G-F 20 appeared to be safe, effective in pain relief and improved functional outcomes ${ }^{[8,9]}$. In this study, we wanted to evaluate the superiority of intra-articular HA over steroids in question. Though, very few studies have been done in this perspective ${ }^{[10-14]}$, the results were varied and did have the limitations. We aimed to overcome these limitations and evaluate the hypothesis.

\section{Material and methods}

After the institutional review board and ethical committee clearance, this study was conducted as a randomized, prospective study between July 2012 and April 2015. Patients of 40 years of age and above presenting with symptoms of degenerative arthritis of knee demonstrating radiological evidence of arthritis [Kellgren-Lawrence (K-L) ${ }^{[15]}$ grade of less than III] and those who were not responding to conservative treatment were taken into the study. Patients with secondary osteoarthritis (gout, pseudogout, septic, tubercular, rheumatoid and psoriasis), degenerative arthritis (K-L > III), unwilling and poor skin condition patients were excluded.

The included patients were subjected to initial evaluation by two separate investigators and subjected to randomization (allocation by alternate numbers). Patients in the steroid group (group 1, MPG) were subjected to two sittings of intraarticular 6-Methylprednisolone acetate 40mg each (day 0 and day 7). Patients in viscosupplementation group (group 2, HG) were given a single intra-articular injection of hylan G-F 20. Both groups were given standard physiotherapy regimen postinjection.

\section{Outcome measure}

We used the following parameters to measure outcome, 1) 100 $\mathrm{mm}$ visual analog pain scale (VAS), 2) Western Ontario and McMaster Universities Osteoarthritis index (WOMAC), 3) SF36 health survey and 4) Range of motion (ROM) to document the baseline characteristics and subsequent follow ups at $2^{\text {nd }}$, $4^{\text {th }}\left(1^{\text {st }}\right.$ month $), 12^{\text {th }}\left(3^{\text {rd }}\right.$ month $), 24^{\text {th }}\left(6^{\text {th }}\right.$ month $)$ and $52^{\text {nd }}(12$ month)week.

72 patients with osteoarthritis were taken into consideration and evaluated. 4 were excluded in view of uncontrolled comorbidity $(n=2)$ and poor skin $(n=2)$ conditions and rest randomized into two groups. 34 patients received 6methylprednisolone acetate and 34 received hylan G-F 20 injection. 3 patients lost to follow up in MPG and 4 did not turn up in HG. Computing was done for the rest. For purposes of analysis, since two knees in the same patient cannot be analysed statistically as independent specimens, patients who received treatment bilaterally underwent an additional randomization step to determine which knee would be included.

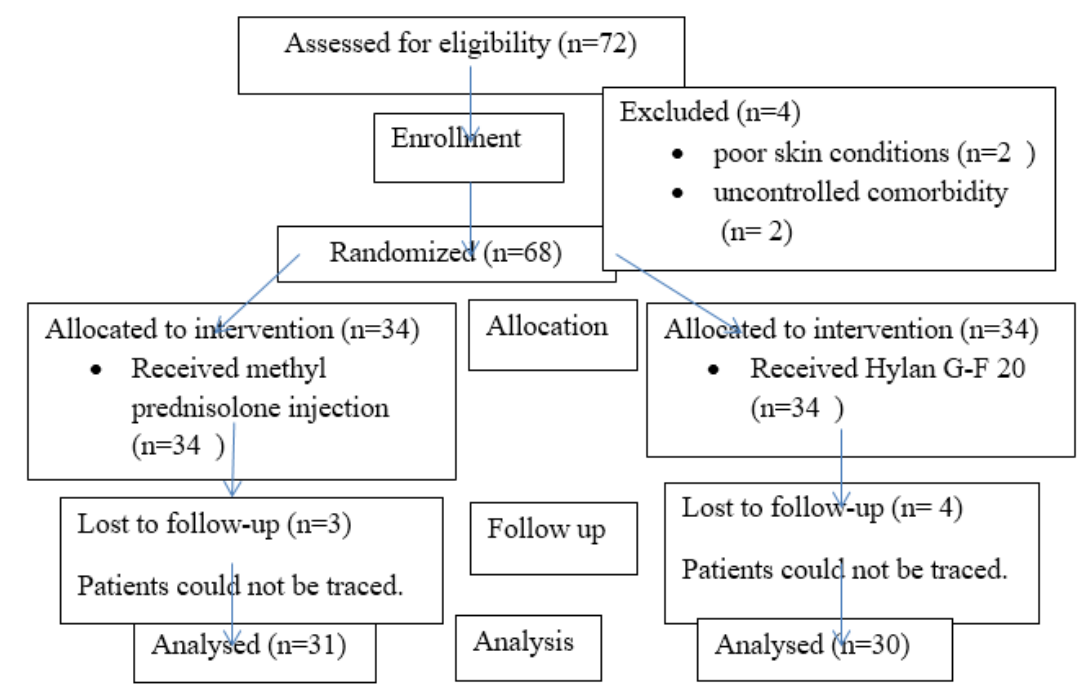

Fig 1: Consort flow chart of events involved in the study

\section{Statistical method}

Statistical testing was conducted with the statistical package for the social science system version SPSS 17.0. Continuous variables are presented as mean $\pm \mathrm{SD}$, and categorical variables are presented as absolute numbers and percentage. The comparison of normally distributed continuous variables between the groups was performed using Student's $t$ test. Paired $t$ test were used for comparison of continuous variables within the groups from post-treatment to pre- treatment. Nominal categorical data between the groups were compared using Chi-squared test or Fisher's exact test as appropriate.

\section{Results}

No statistically significant differences found regarding baseline demographic patterns of patients between two groups. Mean age of patients in group 1 was 56.57 years and in group 
2 was 59.83 years. Female patients account for $54.84 \%$ in group 1 and $53.3 \%$ in group 2. Mean BMI were $25.41 \mathrm{~kg} / \mathrm{m}^{2}$ in group 1 and $26.71 \mathrm{~kg} / \mathrm{m}^{2}$ in group2. $32.3 \%$ patients in group 1 and $43.3 \%$ patients in group 2 have both knee involvements. $36.7 \%$ patients in group 2 have right knee involvement compared to $35.5 \%$ in group 1 . Most of the patients in this study have grade II (K-L grading) radiologic severity of affected knee (48.4\% in group 1 and $63.3 \%$ in group 2$)$. The mean duration of symptoms in group 1 and group 2 were 3.4 years and 3.8 years respectively.

Table 1: Demographic characteristics of study patients

\begin{tabular}{|c|c|c|c|}
\hline Parameters & Group 1(MPG) N=31 & Group 2(HG) N=30 & p value \\
\hline $\begin{array}{c}\text { 1)Age in yrs } \\
\text { (mean } \pm \text { SD) }\end{array}$ & $56.57 \pm 11.08$ & $59.83 \pm 10.73$ & 0.251 \\
\hline $\begin{array}{c}\text { 2)Sex } \\
\text { Male } \\
\text { Female }\end{array}$ & $14(45.16 \%)$ & $14(46.7 \%)$ & 0.438 \\
\hline $\begin{array}{c}\text { 3)BMI in kg/m } \\
\text { (mean } \pm \text { SD) }\end{array}$ & $25.41 \pm 3.07$ & $26.71 \pm 3.14$ & 0.111 \\
\hline 4)Side of knee & $17(53.3 \%)$ & \\
Both & $10(32.3 \%)$ & $13(43.3 \%)$ & 0.422 \\
Left & $10(32.3 \%)$ & $6(20.0 \%)$ & \\
Right & $11(35.5 \%)$ & $11(36.7 \%)$ & 0.129 \\
\hline 5)OA grade & $6(19.4 \%)$ & $1(3.3 \%)$ & $19(63.3 \%)$ \\
I & $15(48.4 \%)$ & $10(31.7 \%)$ & 0.143 \\
\hline
\end{tabular}

Table 2: ROM of knee

\begin{tabular}{|c|c|c|c|c|c|}
\hline ROM & Group 1 (n=31) & & Group 2 (n=30) & & p value \\
\hline & Mean \pm SD & Min-Max & Mean \pm SD & Min-Max & \\
\hline Baseline & $118.33^{\circ} \pm 9.41$ & $90^{\circ}-130^{\circ}$ & $116.50^{\circ} \pm 12.12$ & $90^{\circ}-135^{\circ}$ & 0.515 \\
\hline $54 \mathrm{WK}$ & $120.83^{\circ} \pm 6.25$ & $110^{\circ}-135^{\circ}$ & $126.93^{\circ} \pm 5.29$ & $118^{\circ}-135^{\circ}$ & 0.001 \\
\hline
\end{tabular}

There was no statistically significant difference in the baseline knee range of movements between two groups. From table 2, we can infer that significantly greater improvement of ROM was found in Hylan G-F 20 group at final follow-up when compared to patients who received steroids $(\mathrm{p}=0.001)$. This could be explained by our examination that that the patients after receiving hylan were able to undergo better physiotherapy.

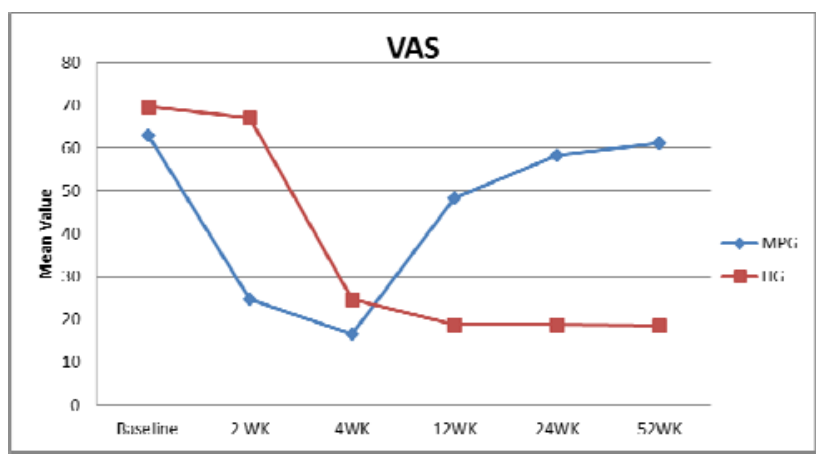

Fig 1: The line diagram of the VAS scores in both groups. Patients instilled with steroids showing decreased patterns beyond 4 weeks. Hylan G-F 20 group show sustained effects.

Statistically no significant differences of baseline VAS score found between the two groups. Patients had significantly better pain relief after methylprednisolone injection at the end of second week and fourth week (VAS score in steroid and hylan group at $2^{\text {nd }}$ week $24.80 \pm 11.31$ and $67.10 \pm 13.97$ respectively, and at $4^{\text {th }}$ week $16.57 \pm 7.21$ and $24.80 \pm 12.26$ respectively) with $\mathrm{p}$ value $<0.05$. But on subsequent follow up, it was examined that the sustained pain effect deteriorated in methylprednisolone group. Pain relief started late in hylan group and sustained till the end of follow up. At the $6^{\text {th }}$ month and $12^{\text {th }}$ month end hylan group patients had significant pain improvements (VAS score in hylan group at $6^{\text {th }}$ and $12^{\text {th }}$ month $18.80 \pm 11.30$ and $18.67 \pm 8.11$ respectively; VAS score in steroid group at $6^{\text {th }}$ and $12^{\text {th }}$ month $58.33 \pm 12.30$ and $61.18 \pm 9.32$ respectively), as compared to methylprednisolone $(\mathrm{p}<0.001)$.

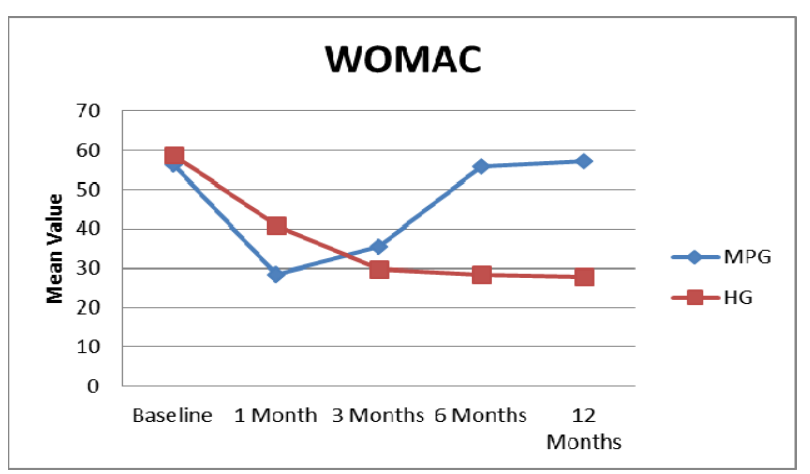

Fig 2: Correlation of WOMAC scores among both the groups suggesting a more sustained pattern of improvement in hylan GF 20 group.

Corticosteroid injected patients showed statistically significant improvement of mean WOMAC score from baseline at 1 month and 3 month $(p<0.05)$. But at month 6 and month 12 , no significant differences found from baseline (baseline, $6^{\text {th }}$ and $12^{\text {th }}$ month mean WOMAC score56.33 \pm 10.86 , $55.93 \pm 13.29$ and $57.12 \pm 8.12$ respectively). Following hylan GF 20 injection patients reported statistically significant and sustained improvement of mean WOMAC score (40.93 \pm 10.27 at 1 st month, $29.67 \pm 7.68$ at 3rd month, $28.43 \pm 11.40$ at 6 th month and $27.97 \pm 8.21$ at $12^{\text {th }}$ month) from baseline (58.83 \pm 9.29$)$. At 1st month mean improvement of WOMAC score was more in corticosteroid group compared to hylan 
treated group $(p<0.001)$. However, score declined gradually in corticosteroid treated group and improved more in hylan treated group at $3^{\text {rd }}, 6^{\text {th }}$ and $12^{\text {th }}$ month with statistically significant differences favoring hylan G-F $20(p<0.05))$.

Similarly, no significant differences found between baseline mean scores of SF 36 functional categories. However, posttreatment SF-36 health survey at final follow-up revealed that, patients in hylan group showed greater improvement of mean score from baseline in all 8 functional categories [physicalfunctioning(PF), role-physical(RP), role-emotional(RE), vitality(VT), social functioning(SF), bodily pain(BP), mental health(MH), and general health $(\mathrm{GH})]$ compared to corticosteroid group ( $\mathrm{p}$ value $<0.001)$.

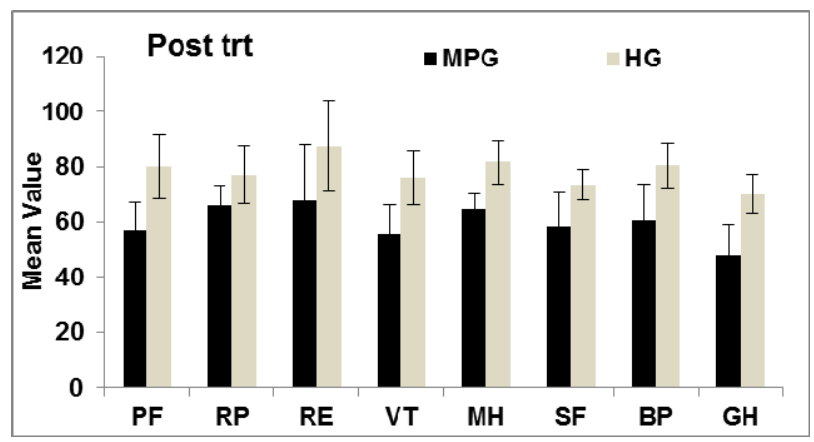

Fig 3: Bar diagram of SF-36 health survey at $6^{\text {th }}$ month posttreatment demonstrating significant functional improvement in hylan group compared to corticosteroid group.

Except for a few raised blood glucose levels post steroid injection, there were no adverse local or systemic effects noted in both groups.

\section{Discussion}

Osteoarthritis is by far the most common form of arthritis and the major cause of disability and reduced activity in people older than 50 yrs. No specific therapy has yet been clearly shown to prevent the progression of OA. The goal is to suppress pain and improve the function. Therapy is individualized on the basis of the symptoms and severity of the disease.

Physicians are increasingly considering intra-articular treatment when simple measures are inadequate. Intra-articular corticosteroids have been used for decades in OA. This treatment provides striking relief of pain especially in patients with synovitis associated with chronic knee OA. New agents called Hyaluronans are also given by intra-articular injection, which serve as a lubricant and shock absorber.

Several studies have compared efficacy and safety of intraarticular hyaluronic acid versus intra-articular corticosteroids. A prospective, randomized trial conducted by Seth S.Leopold et al ${ }^{[12]}$ reported that no differences were detected between patients treated with intra-articular injection of hylan G-F 20 and those treated with corticosteroid with respect to pain relief or function at six months of follow up. In a prospective, randomized study, jones et al ${ }^{[13]}$ reported that patients who received hyaluronic acid injections for the treatment of inflammatory knee arthritis had less pain at six months of follow-up than did patients who received the corticosteroid triamcinolone hexacetonide. Only sixty-three patients were enrolled in that study, and forty-three (68.3\%) of them withdrew most commonly because of worsening of the symptoms-before the six-month final follow-up evaluation. In addition, when those authors applied an intent-to-treat analysis that included all of the patients who had withdrawn, no significant differences were detected between the group treated with hyaluronic acid and that treated with corticosteroid. Outcome assessment in this study was limited to visual analog scale.

Our study evaluated the efficacy of a single intra-articular hylan G-F 20 injection in symptomatic osteoarthritis (K-L < III) and compared it with intra-articular 6-methylprednisolone acetate injection with the outcomes being measured by VAS, WOMAC and SF - 36 scores. The analysis revealed that on a short term basis, both hylan G-F 20 and 6-MP are efficacious in controlling the symptoms related to knee OA. In the corticosteroid group, VAS score showed significant improvement as early as week 2, reaching a peak at week 4, but beyond that at week 12 and week 24 and week 52, this improvement was either mild or absent. In the hylan G-F 20 group, symptoms improved more slowly but progressively and continued to do so after the end of treatment. Significant improvement of VAS score noted at week 4 and it persisted \& in some even improved at week 12 and week 24 and week 52. Recovery of ROM of treated knee was significantly greater in hylan G-F 20 treated group compared to corticosteroid group. Other efficacy variable like WOMAC score also showed significant improvement at week 12 and week 24 in favour of hylan G-F 20 group than that of corticosteroid group. Posttreatment functional outcome measured by SF 36 health survey also showed significant improvement from baseline in all functional categories in hylan G-F 20 group than that of corticosteroid group. Both treatments are well tolerated as none of the patients reported any local or systemic adverse reactions. Our findings were also supported by other studies [10-11, 14].

Randomized clinical trials by Leardini et al ${ }^{[14]}$, in which hyaluronic acid (HA) was compared with a corticosteroid (6methylprednisolone acetate), showed that on a short-term basis, both HA and 6-MP are efficacious in controlling the symptoms related to osteoarthritic disorders. In the long-term assessment, some differences emerged between the two treatments, particularly on the $35^{\text {th }}$ and $60^{\text {th }}$ days when, in the HA-treated group, the results obtained at the end of treatment still persisted and in some cases had been improved. In the 6MP group, on the other hand, painful symptoms returned as early as the $35^{\text {th }}$ day. However the study included a total of only forty patients, the follow up period was limited to two months, and apart from visual analog scale no validated outcome instruments were used. In a multicenter study comparing the activity and tolerability of sodium hyaluronate (HA) and 6-methylprednisoloneacetate (6-MP), V. Pietrogrande et al ${ }^{[10]}$ reported considerable difference between the groups regarding painful symptoms: in the 6-MP group parameters used to assess pain showed a noticeable improvement as early as the seventh and $14^{\text {th }}$ days, reaching a peak between the $28^{\text {th }}$ and $35^{\text {th }}$ days; on the $60^{\text {th }}$ day this improvement was either stable or decreased. In the HA group symptoms improved more slowly but progressively and continued to do so after the end of treatment. The differences between the two groups on the $60^{\text {th }}$ day was in favor of the HA group for all parameters, with statistical significance for spontaneous pain intensity, rest pain, pain under load, morning stiffness, and joint flexion. These observations were also supported by Raveendhara R. Bannuru et al ${ }^{[11]}$.

Hylan GF 20 treated group demonstrated long term benefits in all outcome parameters compared to corticosteroid treated group. A possible explanation for long term efficacy of HA is that exogenous HA intra-articularly injected could stimulate the synthesis of suitable concentration of endogenous, high 
molecular weight HA as demonstrated on synoviocyte culture by smith and ghosh ${ }^{[16]}$. High molecular weight HA not only improve the rheological characteristics of synovial fluid, but also can control some stages of the inflammatory process, acting as a free radicals scavenger [17], inhibiting the chemotaxis of activated macrophages and leukocyte ${ }^{[18]}$, and reducing neutrophils phagocytosis and subsequent enzymatic release ${ }^{[19]}$. Moreover, some studies on experimental model of OA [21], have shown that HA is able to stimulate the intrinsic repair activity of chondrocyte.

The need for a large sample size was a major limitation in our study, others being lack of a placebo control group to compare and correlate, there was no radiological methods used for outcome analysis and the follow up period was only for 12 months. A longer period of follow up was required to evaluate the need and efficacy of repeated hylan injections. The post injection period was not controlled for subject activity level, use of physical therapy or assistive devices, or use of NSAID after injections. These issues must be addressed in future studies to help determine the best overall treatment plan for these patients.

Our study was one of the few studies to evaluate and compare the efficacy of a single intra-articular hylan G-F 20 injection with 6-methylprednisolone acetate in symptomatic osteoarthritis knee patient and also assess it with varied functional subjective and objective scores(VAS, WOMAC and $\mathrm{SF}$ - 36health survey), which was the limitation in various other published studies.

\section{Conclusion}

Intra-articular corticosteroids are fast acting symptomatic drug in OA. Due to the short lived effect of IA steroid, the frequent need for repeated injections limits the usefulness of this agent in long term management of OA. Therefore, their use should be logically focused on short term treatment of disease flares like exacerbation of pain, effusion. Short term effect of intraarticular corticosteroid might be beneficial for those patients, when arthroplasty is deferred due to some unavoidable reasons, to give temporary relief. Whereas, IA hylan G-F 20 acts as slow acting drug with a delayed onset of action (2-4 weeks), and a long lived benefit on pain and function. HA could be used not only to treat symptoms but also as a basic non-surgical therapeutic option for OA, especially in early stage of the disease.

\section{References}

1. Duncan R, Peat G, Thomas E, Hay EM, Croft P. Incidence, progression and sequence of development of radiographic knee osteoarthritis in a symptomatic population. Ann Rheum Dis. 2011; 70(11):1944-8.

2. Howell DS. Pathogenesis of osteoarthritis. Am J Med. 1986; 28:80(4B):24-8.

3. Bijlsma JW, Berenbaum F, Lafeber FP. Osteoarthritis: an update with relevance for clinical practice. Lancet. 2011; 18:377(9783): 2115-26.

4. Zhang W, Nuki G, Moskowitz RW, Abramson S, Altman $\mathrm{RD}$, Arden NK, et al. POARSI recommendations for the management of hip and knee osteoarthritis: part III: Changes in evidence following systematic cumulative update of research published through January 2009. Osteoarthritis Cartilage. 2010; 18(4):476-99.

5. Uthman I, Raynauld JP, Haraoui B. Intra-articular therapy in osteoarthritis. Postgrad Med J. 2003; 79(934):449-53.

6. Habib GS, Saliba W, Nashashibi M. Local effects of intraarticular corticosteroids. Clin Rheumatol. 2010; 29(4):347-56.
7. Habib GS. Systemic effects of intra-articular corticosteroids. Clin Rheumatol. 2009; 28(7):749-56.

8. Goorman SD, Watanabe TK, Miller EH, Perry C. Functional outcome in knee osteoarthritis after treatment with hylan G-F20: a prospective study. Arch Phys Med Rehabil. 2000; 81(4):479-83.

9. Chevalier X, Jerosch J, Goupille P, van Dijk N, Luyten FP, Scott DL et al. Single, intra-articular treatment with 6 ml hylan G-F 20 in patients with symptomatic primary osteoarthritis of the knee: a randomised, multicentre, double-blind, placebo controlled trial. Ann Rheum Dis. 2010; 69(1):113-9.

10. Pietrogrande V, Melanotte PL, D'Agnolo B, Ulivi M, Benigni GA, Turchetto L et al. Hyaluronic acid versus Methylprednisolone intra-articularly injected for treatment of osteoarthritis of the knee. Current therapeutic research. 1991; 50(5):691-701.

11. Bannuru RR, Natov NS, Obadan IE, Price LL, Schmid $\mathrm{CH}$, McAlindon TE. Theraputic trajectory of hyaluronic acid versus corticosteroids in the treatment of knee osteoarthritis: A systemic review and meta-analysis. Arthritis Rheum. 2009 Dec 15; 61(12):1704-11.

12. Leopold SS, Redd BB, Warme WJ, Wehrle PA, Pettis PD, Shott S. Corticosteroid compared with hyaluronic acid injection for the treatment of osteoarthritis of the knee. A prospective, randomized trial. J Bone Joint Surg Am. 2003; 85-A(7):1197-203.

13. Jones AC, Pattrick M, Doherty S, Doherty M. Intraarticular hyaluronic acid compared to intra-articular triamcinolone hexacetonide in inflammatory knee osteoarthritis. Osteoarthritis Cartilage. 1995; 3(4):269-73.

14. Leardini G, Mattara L, Franceschini M, Perbellini A. Intra-articular treatment of knee osteoarthritis. A comparative study between hyaluronic acid and 6methylprednisolone acetate. Clin Exp Rheumatol. 1991; 9(4):375-81.

15. Kellgren JH, Lawrence JS. Radiological Assessment of Osteo-Arthrosis. Ann Rheum Dis. 1957; 16(4):494-502.

16. Smith MM, Ghosh P. The synthesis of hyaluronic acid by human synovial fibroblast is influenced by the nature of hyaluronate in the extracellular environment. Rheumatol Int. 1987; 7(3):113-22.

17. Sato H, Takahashi T, Ide H, Fukushima T, Tabata $\mathrm{M}$, Sekine F et al. Antioxidant activity of synovial fluid, hyaluronic acid, and two subcomponents of hyaluronic acid: synovial fluid scavenging effect is enhanced in rheumatoid arthritis patients. Arthritis Rheum. 1988; 31(1):63-71.

18. Forrester JV, Balazs EA. Inhibition of phagocytosis by high molecular weight hyaluronate. Immunology. 1980; 40(3):435-46.

19. Pisko EJ, Turner RA, Soderstrom LP, Panetti M, Foster SL, Treadway WJ. Inhibition of neutrophils phagocytosis and enzyme release by hyaluronic acid. Clin Exp Rheumatol. 1983; 1(1):41-4.

20. Schiavinato A, Lini E, Guidolin D, Pezzoli G, Botti P, Martelli $\mathrm{M}$ et al. Intra-articular sodium hyaluronate injections in the pond-nuki experimental model of osteoarthritis in dogs.II. Morphological findings. Clin Orthop Relat Res. 1989; (241):286-99.

21. Abatangelo G, Botti P, Del Bue M, Gei G, Samson JC, Cortivo $\mathrm{R}$ et al. Intra-articular sodium hyaluronate injections in the pond-nuki experimental model of osteoarthritis in dogs. I. Biochemical results. Clin Orthop Relat Res. 1989; (241):278-85. 